\title{
Analysis of lipid distribution characteristics in a health examination population in Chengdu (2009-2017)
}

\section{Shanshan Liang}

Department of laboratory medicine, West China hospital, Sichuan University, https://orcid.org/0000-0001-6276-0265

\section{Yanhua Zhao}

west china hospital sichuan university

\section{He Zhang}

west china hospital sichuan university

\section{Wei Gan}

Sichuan University West China Hospital

Guixing Li ( $\nabla$ liguixing27@163.com )

West China hospital, Sichuan University https://orcid.org/0000-0003-0107-213X

\section{Research}

Keywords: blood lipid distribution, health examination population, dyslipidemia

Posted Date: December 23rd, 2020

DOI: https://doi.org/10.21203/rs.3.rs-131509/v1

License: (-) (i) This work is licensed under a Creative Commons Attribution 4.0 International License. Read Full License 


\section{Abstract}

Background

The prevalence of cardiovascular disease is rapidly increasing in the recent years. Dyslipidemia associated atherosclerosis and other complications are posing a serious threat to human health. The present study aimed to analyze the blood lipids distribution characteristics within a large size of health examination population of Sichuan province, China.

Method

This was a retrospective study conducted in 888,143 subjects (495,599 males and 392,544 females) aged more than 18 years old from the Health Examination Center at West China Hospital, Sichuan University during 2009 to 2017. The blood lipids levels were compared in different age groups and different years.

Result

The blood lipids levels were significantly increased in recent years. The percentage of cases with high lipids levels was significantly higher in males than that in females since 2009 to 2017 . Moreover, the blood lipids levels and the percentage of dyslipidemia in aged 51-60 individuals were significantly higher than those in other population every year.

Conclusion

The health examination population showed increased percentage of blood lipids levels, and so regular physical examination and lipids control are highly important in aged population.

\section{Introduction}

High levels of low-density lipoprotein cholesterol (LDL-C) and triacylglycerides have been identified as causal risk factors for atherosclerotic cardiovascular disease[1, 2]. With economic development and cultural changes, the improvement of people's living standard and the change of life style, lead to the gradual increase of blood lipid level of Chinese people, and the prevalence of dyslipidemia. Elevated total cholesterol (TC) and low density lipoprotein cholesterol (LDL-C) are independent risk factors for coronary heart disease and ischemic stroke.

Dyslipidemia refers to abnormal lipid levels in the blood, usually manifested by increased total cholesterol (TC), triglycerides (TGs) and/or low-density lipoprotein cholesterol (LDL) and decreased high-density lipoprotein cholesterol (HDL) [3]. Dyslipidemia is a major risk factor for cardiovascular and cerebrovascular diseases.

Early detection, diagnosis and treatment are important for the prevention of cardiovascular disease and other complications. Regular health examination is an important means for early detection of dyslipidemia. Therefore, the purpose of this study was to analyze blood lipids levels in Sichuan province from 2009 to 2017, and to explore the distribution characteristics of blood lipids in healthy physical examination population, so as to control blood lipid levels.

\section{Methods}

\section{Study design}

The following methods were carried out in accordance with the approved guidelines. A total of 888,143 cases more than 18 years old during 2009 to 2017 , included 495,599 males and 392,544 females were recruited from the Health Examination Center at West China Hospital, Sichuan University. Peripheral blood was collected from all participants. The corresponding protocol was approved by the Ethics of Research Committee of the West China Hospital, Sichuan University. Informed and written consent was obtained from all participating subjects.

\section{Collection of laboratory data and lipids measurement}

Data about demographic and laboratory features were collected from hospital records or by questionnaire. The blood samples were withdrawn from the antecubital vein of subjects in fasting status, and the level of blood lipids level of each individual were detected (cobas 8000 , ROCHE, Germany). Subjects with TGs levels between 0.29 and $1.83 \mathrm{mmol} / \mathrm{L}$ were considered as normal, while with levels $>1.83 \mathrm{mmol} / \mathrm{L}$ were considered as abnormally high. TC between $2.8 \mathrm{mmol} / \mathrm{L}$ and $5.7 \mathrm{mmol} / \mathrm{L}$ were considered as normal, similarly LDL-C<4.0 mmol/L HDL-C $>0.9 \mathrm{mmol} / \mathrm{L}$ were considered as normal. Dyslipidemia was defined as follows: TGs $\geq 1.83 \mathrm{mmol} / \mathrm{L} ; \mathrm{TC} \geq 5.7 \mathrm{mmol} / \mathrm{L} ; \mathrm{LDL}-\mathrm{C} \geq 4.0 \mathrm{mmol} / \mathrm{L}$; and HDL-C $<0.9 \mathrm{mmol} / \mathrm{L}$. Sample collection and laboratory testing were carried out in accordance with the standard operating procedure. Internal quality control data were obtained during the study to ensure the reliability of blood lipids measurement results. Strict calibration and preventive maintenance on the instruments were carried out by the laboratory every month. The programs of the College of American Pathologists external quality assessment were used to ensure the accuracy and reliability of the results.

\section{Statistical analysis}

All statistical analyses were done by Excel 2016 and SPSS 20.0. Before analyzing the data, Excel 2016 was used to remove the values with more than 4 standard deviations $( \pm 4$ SD). A P-value of less than 0.05 was considered to be statistically significant. The results are expressed as medians and values of 
25 to 75 percentiles. Statistical significance of the data was measured by Wilcoxon test for non-normally distributed ones and Chi-square test was used for categorical variables.

\section{Results}

\section{Characteristics of lipids in health examination population}

In the present study, a total of 888,143 cases aged greater than 18 years (495,599 males and 392,544 females) were recruited from 2009 to 2017 . The percentage of males with normal blood lipids level was lower than that in females (Table 1). Health examination in this population revealed that the levels of HDL-C in the year 2009 were $1.41(1.17,1.68) \mathrm{mmol} / \mathrm{L}$, and these were increased by $1.48(1.23,1.79) \mathrm{mmol} / \mathrm{L}$ from 2009 to 2014 . In 2015 to 2017 , the HDL-C levels have been decreased by $1.38(1.13,1.67) \mathrm{mmol} / \mathrm{L}$ (Table 2$)$.

\section{The characteristics of lipids levels between males and females}

Among the health examination population, the lipids levels (cholesterol triglyceride and LDL-C) in different years (2009-2017) were higher in males than that of females, which was the opposing situation of HDL-C level (Table 2).

Among the cases with high lipids(cholesterol $>5.7 \mathrm{mmol} / \mathrm{L}$, triglyceride $>1.83 \mathrm{mmol} / \mathrm{L}$ and $\mathrm{LDL}-\mathrm{C}>4 \mathrm{mmol} / \mathrm{L}$ ) levels and $\mathrm{HDL}<0.9 \mathrm{mmol} / \mathrm{L}, \mathrm{males}$ individuals had a more obvious trend especially at LDL-C and HDL-C (Fig. 1). Moreover, the percentages of males with high lipids (cholesterol>5.7, triglyceride $>1.83$ and LDL-C>4) levels and with lower HDL-C $(H D L-C<0.9)$ were significantly higher than those in females every year $($ all $P<.001)$.

\section{The characteristics of lipids levels among different ages}

Regarding the lipids levels among different ages, the population was divided based on age into 7 groups. The levels of cholesterol were 4.3(3.83, $4.82) \mathrm{mmol} / \mathrm{L}$ in individuals $<30$ years old, and were $5.07(4.5,5.68) \mathrm{mmol} / \mathrm{L}$ in 51 to 60 age group. The trend that individuals $<30$ years old had the lowest lipids levels and the ages group(51-60) had the highest level appeared in the three lipids(cholesterol, triglyceride and LDL-C) level, which was the opposite phenomenon of the HDL-C level (Table 3).

The lipids (cholesterol triglyceride and LDL-C) levels were higher in aged 51-60 individuals of males and 61-70 of females than others' age group every year. People aged 41-50 had the lower HDL-c level than others from 2009-2017 (Table 4). The tendency of lipids levels in different age groups remained the same in different years (Fig2). The percentage of high lipids(cholesterol, triglyceride and LDL-C) levels were significantly higher in 41-50 population of males and 51-60 of females when compared to those in other individuals, and also the percentage of males was higher than females (Fig. 3).

\section{Discussion}

The present study included 888,143 individuals aged 18 to 96 undergoing health examination in the West China Hospital, Sichuan University during 2009 to 2017. This large sample data showed high blood lipids distribution and high percentage of lipids levels in both males and females in the local population. All samples were measured in the West China Hospital, Sichuan University to support the accuracy and stability of the results.

This study showed that the levels of blood lipids were increased year by year in both males as well as females, and similarly the percentage of high lipids levels was also increased from 2009 to 2017 . Compared to women, men had lower levels of HDL-C and significantly higher levels of total cholesterol, LDL-C, triglycerides. This finding was similar to that of the previous study, in which a high incidence of dyslipidemia was observed in males than in females[4-6]. Men may be a risk factor for dyslipidemia. This phenomenon may be caused by excessive accumulation of fat, which is caused by considerable stress and lack of adequate exercise, since men are the main labor force in society. In addition, men smoke and drink more and to a greater degree than women, both of which are linked to dyslipidemia. Moreover, estrogen leads to a reduction in LDL cholesterol and triglycerides, providing relative protection against coronary heart disease in young women[7].

We found that the ages group(51-60) had the highest level appeared in the three lipids(cholesterol, triglyceride and LDL-C) level, which was the opposite phenomenon of the HDL-C level. People aged < 60 would take more high-calorie, high-fat, high-sugar diets than the older individuals. Moreover, the intensity and frequency of exercise among people between the ages of 51 and 60 are lower than that of younger people. Thirdly, study from Ndahimana et al[8] Suggested that older individuals have lower resting energy expenditure compared to younger people. The above three factors make people aged $51-60$ eat more fat and consume less fat, which leads to the occurrence of dyslipidemia.

With rapid economic growth and associated industrialization, urbanization, and lifestyle changes (high-calorie, high-fat, high-sugar diets and decreased physical activity), CVD has reached epidemic proportions in Chinese population. In our study, the percentage of population with high levels of blood lipids was increased in both males and females year by year up to 2015. It is estimated that the number of cardiovascular and cerebrovascular diseases in China is 290 million, including 13 million patients with stroke and 11 million patients with coronary atherosclerotic heart disease (CHD). In the past 20 years, the age-standardized prevalence rate of cardiovascular and cerebrovascular diseases has increased by $14.7 \%$. According to World Bank estimates, the number of stroke and coronary heart disease will increase to 31.77 million and 22.63 million respectively by 2030[9]. Zhang et al[4] found that the prevalence of dyslipidemia in the young population in Wenzhou is relatively high. Young people should increase their health promoting behaviors and consider the factors influencing dyslipidemia to lower the prevalence of dyslipidemia. Therefore, regular physical examination is an important method for early detection of abnormal blood glucose levels, which assists in taking active medical measures for correcting the blood lipids levels. 
As the data was obtained through laboratory information system system, this study adopted screening intervention research method in the epidemiological survey to analyze the variations in the trends of blood lipids concentrations in different genders, different age groups and different years. One of the most important reasons for using big data is due to its accuracy of clinical trial results. To ensure the quality of the data that we collected, sample processing was conducted in accordance with the standard operating procedures of the laboratory. To ensure the reliability of the measurement results, the internal quality control data was verified during the study process.

There are major and distinctive strengths in our study including the large sample size and highly standardized sampling method. However, some limitations should be noted. Firstly, our study was a population-based cross-sectional study with no strict follow-up design. The prevalence of dyslipidemia was based on questionnaires and measurements during a single visit, implying that our results could have been affected by recall bias and unmeasured confounding. Secondly, the definition of dyslipidemia in our study is based on the Chinese guidelines for the prevention and treatment of dyslipidemia in adults, and we should compare our results with those obtained in other countries. Further epidemiological studies are needed to obtain more comprehensive information for the development of prevention and control measures. Thirdly, the information regarding diet and exercise of health examination population were missed. The relationship between blood lipids levels and the above related factors was not explored.

In conclusion, dyslipidemia happens more in males than females. The rate of dyslipidemia increased with age but it appears most obvious in aged 51-60. It is necessary to take steps to control lipids levels in adults, especially in males and the aged population of 51-60. The public should pay more attention on early education and intervention to prevent the development of dyslipidemia.

\section{Declarations}

\section{Ethics approval and consent to participate}

The study was approved by the Institutional Ethics Committee of West China Hospital of Sichuan University and complied with Declaration of Helsinki. Written informed consent was obtained from all subjects.

\section{Consent for publication}

Not applicable.

\section{Availability of data and materials}

The datasets generated and analyzed during the current study can be obtained from the laboratory information system (LIS) of the Department of Laboratory Medicine, West China Hospital, Sichuan University, and are also available from the corresponding author on reasonable request.

\section{Competing interests}

The authors declare that they have no competing interests.

\section{Funding}

This study was supported by Sichuan Science and Technology Program (Grant No 2019YFS0320) to Shanshan Liang. The funding source had no role in design, in the collection, analysis, and interpretation of data; in the writing of the manuscript; and in the decision to submit the manuscript for publication.

\section{Authors' contributions}

Designed the experiments: SSL, GXL. Analyzed the data: YHZ. Wrote the paper: SSL, HZ, GXL. Involved in acquisition of analysis data files from cohort database: YHZ, HZ, WG. Contributed to interpretation of results: SSL, GXL. Critically reviewed drafts of the manuscript and made comments to improve clarity: SSL, YHZ, HZ, WG and GXL. All authors read and approved the final manuscript.

\section{Acknowledgements}

We thank Fei Ding for her suggestion on the scientific design.

\section{References}

1. Ference BA, Ginsberg HN, Graham I, et al. Low-density lipoproteins cause atherosclerotic cardiovascular disease, 1: evidence from genetic, epidemiologic, and clinical studies: a consensus statement from the European Atherosclerosis Society Consensus Panel. Eur Heart J. 2017;38: 24592472.

2. Nordestgaard BG. Triglyceride-rich lipoproteins and atherosclerotic cardiovascular disease: new insights from epidemiology, genetics, and biology. Circ Res. 2016;118: 547-563.

3. Zorrilla E, Del Pilar V alencia M, Serrano PA. Evaluation of a screening test for JAMA. (1974) 228:589-90.

4. Zhang H, Kwapong WR, Shao MM, Yan JY, Lin XD, Chen BB, et al. Predictors of the Prevalence of Dyslipidemia and Influencing Factors for Young Health Examination Cohort: A Cross-Sectional Survey. Front Public Health. 2020;8:400. 
5. Kim KY, Park JS. Impact of fish consumption by subjects with prediabetes on the metabolic risk factors: using data in the 2015 (6th) Korea National Health and Nutrition Examination Surveys. Nutr Res Pract. 2018;12(3):233-42.

6. Gasevic D, Frohlich J, Mancini GJ, et al. Clinical usefulness of lipid ratios to identify men and women with metabolic syndrome: a cross-sectional study. Lipids Health Dis. 2014; 10;13:159.

7. Bush TL, Fried LP, Barrett-Connor E. Cholesterol, lipoproteins, and coronary heart disease in women. Clin Chem. 1988;34(8B):B60-70.

8. Ndahimana D, Kim EK. Measurement Methods for Physical Activity and Energy Expenditure: a Review. Clin Nutr Res. 2017; 6(2):68-80.

9. Beijing Hypertension Association; Beijing Diabetes Prevention and Treatment Association; Beijing Research for Chronic Diseases Control and Health Education. Practice Guide for integrated Management of Primary Cardiovascular Diseases 2020[J]. Chinese Journal of the Frontiers of Medical

Science(Electronic Version), 2020,12(08):1-73

\section{Tables}

Table 1. The characteristics of all health examination population and the people with normal lipids levels.

\begin{tabular}{|c|c|c|c|c|c|c|c|c|c|c|c|c|c|c|c|}
\hline \multirow[b]{2}{*}{ ear } & \multicolumn{3}{|c|}{ Cases, $\mathrm{N}$} & \multicolumn{3}{|c|}{ ses with normal Chol, $\mathrm{N}(\%)$} & \multicolumn{3}{|c|}{ ses with normal TG, $\mathrm{N}(\%)$} & \multicolumn{3}{|c|}{ Cases with nomal LDL-C, $\mathrm{N}(\%)$} & \multicolumn{3}{|c|}{ Cases with normal HDL-C, $\mathrm{N}(\%)$} \\
\hline & Male & Female & all & Male & Female & Overall & Iale & Female & $0 \mathrm{x}$ & Male & Female & Overall & Male & Female & Overall \\
\hline 2009 & 25220 & 98 & & & & t) & 7) & & & & & 7) & & & \\
\hline & & & & & & & & & & & & & & & \\
\hline & & & & & & & & & & & & & & & \\
\hline 2012 & 451 & 500 & & & & & & & & & & & & & \\
\hline 20 & 224 & & & & & & & & & & & & & & \\
\hline & & 955 & & & & & & & & & & & & & \\
\hline & & 592 & & & & & & & & & & & & & \\
\hline & & 71. & & & & & & & & & & & & & \\
\hline 2017 & 91526 & 76980 & 1 & & 97) & & 65.62) & .13) & & & & & & & -1 \\
\hline tal & 5599 & 544 & & $9(83.18)$ & $000(85.57)$ & $748139(84.24)$ & $327661(66.11)$ & 345820088.1 & $481(75.83)$ & $34(92.88)$ & $372849(94.98)$ & $833183(93.81)$ & $458778(92.57)$ & 77819 & 3465 \\
\hline
\end{tabular}

Table2: Characteristics of the cases of lipids among the health examination population(2009-2017).

\begin{tabular}{|c|c|c|c|c|c|c|c|c|c|}
\hline & cholesterol & & & & & TG & & & \\
\hline Year & Overall(mmol/L) & Male(mmol/L) & Famale(mmol/L) & $\mathrm{p}$ & Year & Overall(mmol/L) & Male(mmol/L) & Famale(mmol/L) & $\mathrm{p}$ \\
\hline 2009 & $4.62(4.07,5.24)$ & $4.69(4.15,5.29)$ & $4.52(3.97,5.16)$ & $<0.001$ & 2009 & $1.3(0.91,1.95)$ & $1.54(1.09,2.26)$ & $1.03(0.77,1.48)$ & $<0.001$ \\
\hline 2010 & $4.69(4.14,5.31$ & $4.77(4.22,5.37)$ & $4.59(4.04,5.23)$ & $<0.001$ & 2010 & $1.17(0.81,1.75)$ & $1.4(0.98,2.05)$ & $0.92(0.69,1.32)$ & $<0.001$ \\
\hline 2011 & $4.81(4.25,5.45)$ & $4.87(4.31,5.5)$ & $4.73(4.17,5.39)$ & $<0.001$ & 2011 & $1.2(0.84,1.8)$ & $1.43(1.01,2.11)$ & $0.97(0.71,1.37)$ & $<0.001$ \\
\hline 2012 & $4.71(4.15,5.32)$ & $4.78(4.21,5.38)$ & $4.61(4.07,5.24)$ & $<0.001$ & 2012 & $1.2(0.84,1.79)$ & $1.44(1.01,2.13)$ & $0.95(0.71,1.35)$ & $<0.001$ \\
\hline 2013 & $4.74(4.17,5.36)$ & $4.8(4.23,5.41)$ & $4.65(4.09,5.28)$ & $<0.001$ & 2013 & $1.23(0.85,1.83)$ & $1.47(1.03,2.16)$ & $0.98(0.72,1.4)$ & $<0.001$ \\
\hline 2014 & $4.76(4.19,5.38)$ & $4.82(4.25,5.43)$ & $4.68(4.11,5.31)$ & $<0.001$ & 2014 & $1.2(0.84,1.8)$ & $1.45(1.01,2.13)$ & $0.97(0.72,1.39)$ & $<0.001$ \\
\hline 2015 & $4.81(4.24,5.43)$ & $4.88(4.31,5.49)$ & $4.72(4.16,5.35)$ & $<0.001$ & 2015 & $1.23(0.86,1.83)$ & $1.48(1.04,2.16)$ & $0.98(0.72,1.39)$ & $<0.001$ \\
\hline 2016 & $4.78(4.21,5.4)$ & $4.84(4.27,5.45)$ & $4.69(4.13,5.33)$ & $<0.001$ & 2016 & $1.18(0.82,1.76)$ & $1.43(0.99,2.1)$ & $0.94(0.69,1.34)$ & $<0.001$ \\
\hline \multirow[t]{2}{*}{2017} & $4.73(4.17,5.35)$ & $4.79(4.24,5.4)$ & $4.65(4.11,5.28)$ & $<0.001$ & 2017 & $1.21(0.84,1.8)$ & $1.47(1.03,2.16)$ & $0.97(0.72,1.38)$ & $<0.001$ \\
\hline & LDL-C & & & & & HDL-C & & & \\
\hline Year & Overall(mmol/L) & Male(mmol/L) & Famale(mmol/L) & $\mathrm{p}$ & Year & Overall(mmol/L) & Male(mmol/L) & Famale(mmol/L) & $\mathrm{p}$ \\
\hline 2009 & $2.72(2.24,3.25)$ & $2.83(2.35,3.34)$ & $2.56(2.1,3.1)$ & $<0.001$ & 2009 & $1.41(1.17 \rrbracket 1.68)$ & $1.27(1.08,1.5)$ & $1.61(1.38,1.86)$ & $<0.001$ \\
\hline 2010 & $2.68(2.2,3.22)$ & $2.81(2.33,3.32)$ & $2.51(2.07,3.05)$ & $<0.001$ & 2010 & $1.45(1.21 \llbracket 1.74)$ & $1.31(1.12,1.54)$ & $1.65(1.42,1.92)$ & $<0.001$ \\
\hline 2011 & $2.69(2.2,3.22)$ & $2.8(2.31,3.31)$ & $2.54(2.08,3.09)$ & $<0.001$ & 2011 & $1.5(1.25 \bowtie 1.8)$ & $1.35(1.15,1.59)$ & $1.72(1.47,1.98)$ & $<0.001$ \\
\hline 2012 & $2.77(2.28,3.31)$ & $2.9(2.41,3.42)$ & $2.6(2.14,3.14)$ & $<0.001$ & 2012 & $1.45(1.2 \bigotimes 1.75)$ & $1.3(1.1,1.54)$ & $1.67(1.43,1.93)$ & $<0.001$ \\
\hline 2013 & $2.8(2.3,3.35)$ & $2.93(2.43,3.45)$ & $2.64(2.17,3.19)$ & $<0.001$ & 2013 & $1.44(1.2 \otimes 1.74)$ & $1.29(1.1,1.53)$ & $1.66(1.42,1.92)$ & $<0.001$ \\
\hline 2014 & $2.73(2.24,3.27)$ & $2.85(2.36,3.36)$ & $2.58(2.12,3.13)$ & $<0.001$ & 2014 & $1.48(1.23 \llbracket 1.79)$ & $1.32(1.12,1.56)$ & $1.7(1.45,1.98)$ & $<0.001$ \\
\hline 2015 & $2.64(2.17,3.15)$ & $2.75(2.29,3.25)$ & $2.48(2.05,3)$ & $<0.001$ & 2015 & 1.47(1.21区1.77) & $1.31(1.1,1.55)$ & $1.69(1.43,1.97)$ & $<0.001$ \\
\hline 2016 & $2.66(2.19,3.17)$ & $2.77(2.31,3.26)$ & $2.51(2.07,3.02)$ & $<0.001$ & 2016 & $1.39(1.14 \llbracket 1.68)$ & $1.23(1.04,1.46)$ & $1.61(1.36,1.87)$ & $<0.001$ \\
\hline 2017 & $2.78(2.29,3.31)$ & $2.9(2.41,3.41)$ & $2.63(2.17,3.17)$ & $<0.001$ & 2017 & 1.38(1.13囚1.67) & $1.22(1.03,1.44)$ & $1.59(1.35,1.86)$ & $<0.001$ \\
\hline
\end{tabular}

Table 3: The lipids level in different ages of health examination population 


\begin{tabular}{|c|c|c|c|c|c|c|}
\hline \multicolumn{7}{|c|}{ cholesterol } \\
\hline \multirow[t]{2}{*}{ Age } & \multirow[t]{2}{*}{ Number } & Minimum & \multirow[t]{2}{*}{ Percentile $25 \%(\mathrm{mmol} / \mathrm{L})$} & Median & \multirow[t]{2}{*}{ Percentile $75 \%(\mathrm{mmol} / \mathrm{L})$} & \multirow{2}{*}{$\begin{array}{l}\text { Maximum } \\
\text { (mmol/L) }\end{array}$} \\
\hline & & $(\mathrm{mmol} / \mathrm{L})$ & & $(\mathrm{mmol} / \mathrm{L})$ & & \\
\hline$\leq 30$ & 137872 & 0.09 & 3.83 & 4.3 & 4.82 & 13.14 \\
\hline $31-40$ & 222300 & 0.13 & 4.05 & 4.56 & 5.13 & 18.1 \\
\hline $41-50$ & 250344 & 0.15 & 4.3 & 4.83 & 5.41 & 26.85 \\
\hline $51-60$ & 154555 & 0.46 & 4.5 & 5.07 & 5.68 & 15.54 \\
\hline $61-70$ & 70059 & 0.42 & 4.48 & 5.07 & 5.7 & 18.89 \\
\hline $71-80$ & 39795 & 0.44 & 4.34 & 4.97 & 5.63 & 13.31 \\
\hline$>80$ & 13218 & 0.53 & 4.19 & 4.86 & 5.53 & 12.2 \\
\hline
\end{tabular}

\begin{tabular}{|c|c|c|c|c|c|c|}
\hline \multicolumn{7}{|l|}{ TG } \\
\hline \multirow[t]{2}{*}{ Age } & \multirow[t]{2}{*}{ Number } & Minimum & \multirow[t]{2}{*}{ Percentile 25\%(mmol/L) } & Median & \multirow[t]{2}{*}{ Percentile $75 \%(\mathrm{mmol} / \mathrm{L})$} & Maximum \\
\hline & & $(\mathrm{mmol} / \mathrm{L})$ & & $(\mathrm{mmol} / \mathrm{L})$ & & $(\mathrm{mmol} / \mathrm{L})$ \\
\hline$\leq 30$ & 137867 & 0.05 & 0.67 & 0.89 & 1.29 & 36.23 \\
\hline $31-40$ & 22296 & 0.07 & 0.78 & 1.12 & 1.72 & 25.71 \\
\hline $41-50$ & 250336 & 0.02 & 0.91 & 1.31 & 1.98 & 41.07 \\
\hline $51-60$ & 154541 & 0.07 & 1.02 & 1.42 & 2.04 & 26.54 \\
\hline $61-70$ & 70058 & 0.14 & 1.01 & 1.38 & 1.92 & 23.35 \\
\hline $71-80$ & 39794 & 0.12 & 0.97 & 1.31 & 1.79 & 22.77 \\
\hline$>80$ & 13218 & 0.22 & 0.9 & 1.22 & 1.66 & 15.89 \\
\hline
\end{tabular}




\begin{tabular}{|c|c|c|c|c|c|c|c|c|c|c|}
\hline $\begin{array}{l}\text { LDL- } \\
\text { C }\end{array}$ & & & & & & & & & & \\
\hline \multirow{3}{*}{$\begin{array}{l}\text { Age } \\
\leq 30\end{array}$} & \multirow{3}{*}{$\begin{array}{l}\text { Number } \\
137060\end{array}$} & \multirow{2}{*}{$\begin{array}{l}\text { Minimum } \\
\text { (mmol/L) }\end{array}$} & \multirow{2}{*}{\multicolumn{2}{|c|}{ Percentile $25 \%(\mathrm{mmol} / \mathrm{L})$}} & \multirow{2}{*}{\multicolumn{2}{|c|}{$\begin{array}{l}\text { Median } \\
\text { (mmol/L) }\end{array}$}} & \multirow{2}{*}{\multicolumn{2}{|c|}{ Percentile $75 \%(\mathrm{mmol} / \mathrm{L})$}} & \multirow{2}{*}{\multicolumn{2}{|c|}{$\begin{array}{l}\text { Maximum } \\
(\mathrm{mmol} / \mathrm{L})\end{array}$}} \\
\hline & & & & & & & & & & \\
\hline & & 0.02 & \multicolumn{2}{|l|}{1.95} & \multicolumn{2}{|c|}{2.35} & \multicolumn{2}{|l|}{2.82} & \multicolumn{2}{|c|}{10.47} \\
\hline $\begin{array}{l}31- \\
40\end{array}$ & 222298 & 0.04 & \multicolumn{2}{|l|}{2.14} & \multicolumn{2}{|c|}{2.58} & \multicolumn{2}{|l|}{3.08} & \multicolumn{2}{|l|}{11.05} \\
\hline $\begin{array}{l}41- \\
50\end{array}$ & 250341 & 0.03 & \multicolumn{2}{|l|}{2.32} & \multicolumn{2}{|l|}{2.79} & \multicolumn{2}{|l|}{3.29} & \multicolumn{2}{|l|}{11.53} \\
\hline $\begin{array}{l}51- \\
60\end{array}$ & 154553 & 0.02 & \multicolumn{2}{|l|}{2.5} & \multicolumn{2}{|l|}{2.98} & \multicolumn{2}{|l|}{3.5} & \multicolumn{2}{|l|}{12.44} \\
\hline $\begin{array}{l}61- \\
70\end{array}$ & 70058 & 0.09 & \multicolumn{2}{|l|}{2.47} & \multicolumn{2}{|l|}{2.99} & \multicolumn{2}{|l|}{3.51} & \multicolumn{2}{|l|}{10.37} \\
\hline $\begin{array}{l}71- \\
80\end{array}$ & 39794 & 0.25 & \multicolumn{2}{|l|}{2.36} & 2.89 & & 3.45 & & 10.5 & \\
\hline$>80$ & 13217 & 0.2 & 2.18 & & 2.75 & & 3.33 & & 12.21 & \\
\hline $\begin{array}{l}\text { HDL- } \\
\mathrm{C}\end{array}$ & & & & & & & & & & \\
\hline Age & Number & Minimum & & Percentile 25\%(mmol/L) & & Median & & Percentile $75 \%(\mathrm{mmol} / \mathrm{L})$ & & Maximum \\
\hline & & $(\mathrm{mmol} / \mathrm{L})$ & & & & $(\mathrm{mmol} / \mathrm{L})$ & & & & $(\mathrm{mmol} / \mathrm{L})$ \\
\hline$\leq 30$ & 137057 & 0.09 & & 1.27 & & 1.52 & & 1.79 & & 4.04 \\
\hline $\begin{array}{l}31- \\
40\end{array}$ & 222296 & 0.08 & & 1.18 & & 1.43 & & 1.72 & & 4.56 \\
\hline $\begin{array}{l}41- \\
50\end{array}$ & 250340 & 0.02 & & 1.16 & & 1.41 & & 1.71 & & 4.86 \\
\hline $\begin{array}{l}51- \\
60\end{array}$ & 154545 & 0.09 & & 1.17 & & 1.41 & & 1.71 & & 4.62 \\
\hline $\begin{array}{l}61- \\
70\end{array}$ & 70052 & 0.05 & & 1.22 & & 1.45 & & 1.74 & & 4.69 \\
\hline $\begin{array}{l}71- \\
80\end{array}$ & 39795 & 0.15 & & 1.23 & & 1.48 & & 1.79 & & 4.45 \\
\hline$>80$ & 13217 & 0.2 & & 1.28 & & 1.55 & & 1.89 & & 4.98 \\
\hline
\end{tabular}

Table 4-1 The characteristics of cholesterol among the health examination population in different ages (2009-2017). 


\begin{tabular}{|c|c|c|c|c|c|c|c|c|c|}
\hline & & Male & & & & Female & & & \\
\hline YEAR & AGE & $\mathrm{n}$ & p25 & median & p75 & $\mathrm{n}$ & p25 & median & p75 \\
\hline \multirow[t]{7}{*}{2009} & $<30$ & 3335 & 3.75 & 4.24 & 4.78 & 3365 & 3.64 & 4.06 & 4.53 \\
\hline & $30-40$ & 6016 & 4.09 & 4.6 & 5.18 & 4540 & 3.79 & 4.22 & 4.69 \\
\hline & $40-50$ & 6843 & 4.26 & 4.8 & 5.38 & 4881 & 4.08 & 4.57 & 5.11 \\
\hline & $50-60$ & 4530 & 4.32 & 4.85 & 5.43 & 2858 & 4.52 & 5.09 & 5.69 \\
\hline & $60-70$ & 2410 & 4.29 & 4.82 & 5.43 & 1494 & 4.66 & 5.22 & 5.78 \\
\hline & $70-80$ & 1666 & 4.24 & 4.8 & 5.44 & 902 & 4.71 & 5.27 & 5.88 \\
\hline & $>80$ & 420 & 4.21 & 4.78 & 5.35 & 158 & 4.68 & 5.37 & 5.94 \\
\hline \multirow[t]{7}{*}{2010} & $<30$ & 4768 & 3.83 & 4.32 & 4.89 & 5132 & 3.74 & 4.15 & 4.64 \\
\hline & $30-40$ & 8386 & 4.17 & 4.7 & 5.27 & 6793 & 3.87 & 4.33 & 4.81 \\
\hline & $40-50$ & 9919 & 4.35 & 4.89 & 5.48 & 7417 & 4.15 & 4.66 & 5.21 \\
\hline & $50-60$ & 5759 & 4.38 & 4.92 & 5.53 & 3724 & 4.64 & 5.2 & 5.79 \\
\hline & $60-70$ & 2964 & 4.35 & 4.92 & 5.51 & 1946 & 4.76 & 5.33 & 5.95 \\
\hline & $70-80$ & 2055 & 4.29 & 4.81 & 5.47 & 954 & 4.63 & 5.28 & 5.96 \\
\hline & $>80$ & 627 & 4.15 & 4.86 & 5.41 & 181 & 4.68 & 5.31 & 5.94 \\
\hline \multirow[t]{7}{*}{2011} & $<30$ & 4693 & 3.92 & 4.42 & 4.99 & 5014 & 3.83 & 4.27 & 4.74 \\
\hline & $30-40$ & 8683 & 4.25 & 4.78 & 5.38 & 7113 & 3.97 & 4.43 & 4.94 \\
\hline & $40-50$ & 11502 & 4.44 & 4.99 & 5.61 & 8259 & 4.28 & 4.77 & 5.33 \\
\hline & $50-60$ & 6378 & 4.48 & 5.04 & 5.67 & 4242 & 4.75 & 5.32 & 5.92 \\
\hline & $60-70$ & 3164 & 4.4 & 4.96 & 5.59 & 2211 & 4.83 & 5.44 & 6.08 \\
\hline & $70-80$ & 2278 & 4.36 & 4.95 & 5.55 & 1421 & 4.73 & 5.4 & 6.06 \\
\hline & $>80$ & 787 & 4.28 & 4.86 & 5.46 & 293 & 4.65 & 5.3 & 6.04 \\
\hline \multirow[t]{7}{*}{2012} & $<30$ & 6146 & 3.8 & 4.32 & 4.89 & 7157 & 3.77 & 4.18 & 4.66 \\
\hline & $30-40$ & 10361 & 4.2 & 4.73 & 5.31 & 8306 & 3.91 & 4.36 & 4.86 \\
\hline & $40-50$ & 13318 & 4.36 & 4.92 & 5.52 & 9728 & 4.2 & 4.69 & 5.26 \\
\hline & $50-60$ & 7067 & 4.4 & 4.96 & 5.54 & 4490 & 4.67 & 5.23 & 5.82 \\
\hline & $60-70$ & 3314 & 4.31 & 4.85 & 5.47 & 2185 & 4.74 & 5.32 & 5.89 \\
\hline & $70-80$ & 2376 & 4.22 & 4.78 & 5.36 & 1323 & 4.58 & 5.29 & 5.94 \\
\hline & $>80$ & 869 & 4.1 & 4.68 & 5.3 & 311 & 4.47 & 5.08 & 5.92 \\
\hline \multirow[t]{7}{*}{2013} & $<30$ & 6307 & 3.91 & 4.4 & 4.96 & 7327 & 3.78 & 4.21 & 4.7 \\
\hline & $30-40$ & 11869 & 4.18 & 4.73 & 5.32 & 9931 & 3.9 & 4.36 & 4.86 \\
\hline & $40-50$ & 14940 & 4.36 & 4.92 & 5.53 & 11376 & 4.2 & 4.72 & 5.27 \\
\hline & $50-60$ & 9415 & 4.39 & 4.95 & 5.54 & 6287 & 4.65 & 5.21 & 5.81 \\
\hline & $60-70$ & 4295 & 4.31 & 4.9 & 5.46 & 3034 & 4.7 & 5.28 & 5.92 \\
\hline & $70-80$ & 3069 & 4.2 & 4.8 & 5.41 & 1952 & 4.51 & 5.2 & 5.83 \\
\hline & $>80$ & 1129 & 4.04 & 4.7 & 5.3 & 444 & 4.43 & 5.17 & 5.89 \\
\hline \multirow[t]{6}{*}{2014} & $<30$ & 4752 & 3.93 & 4.44 & 4.99 & 5592 & 3.8 & 4.23 & 4.72 \\
\hline & $30-40$ & 8987 & 4.21 & 4.75 & 5.32 & 7878 & 3.94 & 4.4 & 4.9 \\
\hline & $40-50$ & 10554 & 4.39 & 4.94 & 5.54 & 8038 & 4.21 & 4.73 & 5.27 \\
\hline & $50-60$ & 7181 & 4.4 & 4.97 & 5.59 & 5051 & 4.67 & 5.26 & 5.84 \\
\hline & $60-70$ & 3509 & 4.33 & 4.92 & 5.49 & 2633 & 4.64 & 5.24 & 5.87 \\
\hline & $70-80$ & 2201 & 4.2 & 4.79 & 5.39 & 1368 & 4.59 & 5.2 & 5.88 \\
\hline
\end{tabular}

Page 8/18 


\begin{tabular}{|c|c|c|c|c|c|c|c|c|c|}
\hline & $>80$ & 929 & 4.05 & 4.75 & 5.4 & 395 & 4.36 & 5.09 & 5.81 \\
\hline \multirow[t]{7}{*}{2015} & $<30$ & 11113 & 4 & 4.53 & 5.09 & 13104 & 3.86 & 4.29 & 4.8 \\
\hline & $30-40$ & 20778 & 4.27 & 4.8 & 5.39 & 16959 & 4 & 4.45 & 4.98 \\
\hline & $40-50$ & 24382 & 4.45 & 4.99 & 5.6 & 18354 & 4.27 & 4.79 & 5.34 \\
\hline & $50-60$ & 16531 & 4.48 & 5.04 & 5.66 & 10476 & 4.73 & 5.31 & 5.92 \\
\hline & $60-70$ & 6432 & 4.39 & 4.96 & 5.59 & 4740 & 4.77 & 5.34 & 5.98 \\
\hline & $70-80$ & 3768 & 4.22 & 4.86 & 5.47 & 2306 & 4.59 & 5.3 & 5.96 \\
\hline & $>80$ & 1429 & 4.07 & 4.76 & 5.4 & 653 & 4.44 & 5.16 & 5.89 \\
\hline \multirow[t]{7}{*}{2016} & $<30$ & 11509 & 3.96 & 4.47 & 5.04 & 13160 & 3.83 & 4.27 & 4.77 \\
\hline & $30-40$ & 22158 & 4.24 & 4.77 & 5.36 & 18756 & 3.96 & 4.43 & 4.95 \\
\hline & $40-50$ & 25432 & 4.39 & 4.94 & 5.55 & 19691 & 4.25 & 4.76 & 5.31 \\
\hline & $50-60$ & 17980 & 4.43 & 4.99 & 5.61 & 11619 & 4.7 & 5.27 & 5.89 \\
\hline & $60-70$ & 7462 & 4.32 & 4.92 & 5.55 & 5050 & 4.74 & 5.32 & 5.95 \\
\hline & $70-80$ & 3776 & 4.15 & 4.79 & 5.43 & 2311 & 4.58 & 5.23 & 5.94 \\
\hline & $>80$ & 1552 & 4 & 4.7 & 5.37 & 681 & 4.4 & 5.2 & 5.93 \\
\hline \multirow[t]{7}{*}{2017} & $<30$ & 11698 & 3.93 & 4.43 & 5 & 13700 & 3.82 & 4.26 & 4.74 \\
\hline & $30-40$ & 23436 & 4.21 & 4.72 & 5.31 & 21350 & 3.93 & 4.37 & 4.89 \\
\hline & $40-50$ & 25386 & 4.36 & 4.9 & 5.5 & 20324 & 4.21 & 4.72 & 5.27 \\
\hline & $50-60$ & 18155 & 4.37 & 4.94 & 5.55 & 12812 & 4.66 & 5.22 & 5.82 \\
\hline & $60-70$ & 7614 & 4.31 & 4.9 & 5.51 & 5602 & 4.69 & 5.29 & 5.92 \\
\hline & $70-80$ & 3668 & 4.12 & 4.74 & 5.37 & 2401 & 4.43 & 5.15 & 5.85 \\
\hline & $>80$ & 1569 & 3.9 & 4.59 & 5.28 & 791 & 4.33 & 5.07 & 5.77 \\
\hline
\end{tabular}

Table 4-2 The characteristics of triglyceride among the health examination population in different ages (2009-2017). 


\begin{tabular}{|c|c|c|c|c|c|c|c|c|c|}
\hline & & Male & & & & Female & & & \\
\hline YEAR & AGE & $\mathrm{n}$ & p25 & median & p75 & $\mathrm{n}$ & p25 & median & p75 \\
\hline \multirow[t]{7}{*}{2009} & $<30$ & 3335 & 0.87 & 1.2 & 1.74 & 3365 & 0.63 & 0.8 & 1.03 \\
\hline & $30-40$ & 6016 & 1.1 & 1.57 & 2.34 & 4540 & 0.69 & 0.89 & 1.19 \\
\hline & $40-50$ & 6843 & 1.24 & 1.78 & 2.57 & 4881 & 0.8 & 1.04 & 1.43 \\
\hline & $50-60$ & 4530 & 1.17 & 1.63 & 2.33 & 2858 & 0.98 & 1.35 & 1.89 \\
\hline & $60-70$ & 2410 & 1.07 & 1.45 & 2.03 & 1494 & 1.16 & 1.54 & 2.14 \\
\hline & $70-80$ & 1666 & 0.99 & 1.33 & 1.83 & 902 & 1.19 & 1.6 & 2.19 \\
\hline & $>80$ & 420 & 0.94 & 1.22 & 1.74 & 158 & 1.15 & 1.6 & 2.21 \\
\hline \multirow[t]{7}{*}{2010} & $<30$ & 4767 & 0.77 & 1.07 & 1.58 & 5131 & 0.57 & 0.72 & 0.95 \\
\hline & $30-40$ & 8384 & 0.99 & 1.41 & 2.09 & 6794 & 0.63 & 0.81 & 1.08 \\
\hline & $40-50$ & 9919 & 1.11 & 1.6 & 2.35 & 7416 & 0.72 & 0.95 & 1.31 \\
\hline & $50-60$ & 5759 & 1.06 & 1.49 & 2.16 & 3722 & 0.89 & 1.2 & 1.66 \\
\hline & $60-70$ & 2964 & 0.96 & 1.32 & 1.87 & 1946 & 1.03 & 1.41 & 1.96 \\
\hline & $70-80$ & 2055 & 0.9 & 1.2 & 1.63 & 954 & 1.08 & 1.47 & 1.97 \\
\hline & $>80$ & 627 & 0.89 & 1.21 & 1.6 & 181 & 0.98 & 1.33 & 1.79 \\
\hline \multirow[t]{7}{*}{2011} & $<30$ & 4693 & 0.81 & 1.14 & 1.65 & 5014 & 0.6 & 0.75 & 0.97 \\
\hline & $30-40$ & 8683 & 1.01 & 1.44 & 2.17 & 7113 & 0.64 & 0.83 & 1.12 \\
\hline & $40-50$ & 11502 & 1.15 & 1.63 & 2.39 & 8259 & 0.74 & 0.99 & 1.35 \\
\hline & $50-60$ & 6378 & 1.08 & 1.53 & 2.2 & 4242 & 0.91 & 1.23 & 1.73 \\
\hline & $60-70$ & 3164 & 0.97 & 1.35 & 1.89 & 2211 & 1.02 & 1.38 & 1.89 \\
\hline & $70-80$ & 2278 & 0.91 & 1.22 & 1.66 & 1421 & 1.06 & 1.4 & 1.91 \\
\hline & $>80$ & 787 & 0.85 & 1.19 & 1.66 & 293 & 1 & 1.32 & 1.77 \\
\hline \multirow[t]{7}{*}{2012} & $<30$ & 6146 & 0.78 & 1.09 & 1.6 & 7157 & 0.59 & 0.76 & 0.99 \\
\hline & $30-40$ & 10362 & 1.03 & 1.47 & 2.21 & 8306 & 0.66 & 0.84 & 1.13 \\
\hline & $40-50$ & 13318 & 1.16 & 1.65 & 2.4 & 9728 & 0.75 & 0.99 & 1.35 \\
\hline & $50-60$ & 7067 & 1.11 & 1.55 & 2.22 & 4490 & 0.91 & 1.25 & 1.74 \\
\hline & $60-70$ & 3314 & 0.96 & 1.34 & 1.89 & 2185 & 1.05 & 1.4 & 1.92 \\
\hline & $70-80$ & 2376 & 0.9 & 1.23 & 1.69 & 1323 & 1.06 & 1.41 & 1.91 \\
\hline & $>80$ & 869 & 0.86 & 1.15 & 1.55 & 311 & 1.02 & 1.35 & 1.76 \\
\hline \multirow[t]{7}{*}{2013} & $<30$ & 6307 & 0.82 & 1.15 & 1.68 & 7327 & 0.6 & 0.77 & 1.01 \\
\hline & $30-40$ & 11869 & 1.04 & 1.5 & 2.21 & 9931 & 0.66 & 0.85 & 1.14 \\
\hline & $40-50$ & 14940 & 1.17 & 1.66 & 2.44 & 11376 & 0.76 & 1 & 1.38 \\
\hline & $50-60$ & 9415 & 1.11 & 1.57 & 2.26 & 6287 & 0.92 & 1.25 & 1.74 \\
\hline & $60-70$ & 4295 & 1 & 1.38 & 1.92 & 3034 & 1.04 & 1.42 & 1.94 \\
\hline & $70-80$ & 3069 & 0.93 & 1.27 & 1.76 & 1952 & 1.05 & 1.41 & 1.86 \\
\hline & $>80$ & 1129 & 0.85 & 1.16 & 1.57 & 444 & 1.01 & 1.33 & 1.81 \\
\hline \multirow[t]{6}{*}{2014} & $<30$ & 4752 & 0.82 & 1.15 & 1.71 & 5591 & 0.6 & 0.76 & 0.98 \\
\hline & $30-40$ & 8986 & 1.02 & 1.48 & 2.17 & 7878 & 0.66 & 0.85 & 1.15 \\
\hline & $40-50$ & 10554 & 1.14 & 1.65 & 2.41 & 8038 & 0.73 & 0.97 & 1.35 \\
\hline & $50-60$ & 7181 & 1.09 & 1.54 & 2.26 & 5051 & 0.91 & 1.23 & 1.71 \\
\hline & $60-70$ & 3509 & 0.99 & 1.34 & 1.89 & 2633 & 1.03 & 1.4 & 1.93 \\
\hline & $70-80$ & 2201 & 0.9 & 1.21 & 1.67 & 1368 & 1.06 & 1.41 & 1.89 \\
\hline
\end{tabular}

Page 10/18 


\begin{tabular}{|c|c|c|c|c|c|c|c|c|c|}
\hline & $>80$ & 929 & 0.83 & 1.1 & 1.49 & 395 & 0.95 & 1.28 & 1.68 \\
\hline \multirow[t]{7}{*}{2015} & $<30$ & 11113 & 0.85 & 1.18 & 1.72 & 13104 & 0.61 & 0.78 & 1.01 \\
\hline & $30-40$ & 20775 & 1.03 & 1.49 & 2.2 & 16959 & 0.66 & 0.86 & 1.18 \\
\hline & $40-50$ & 24379 & 1.15 & 1.64 & 2.4 & 18353 & 0.76 & 1.01 & 1.39 \\
\hline & $50-60$ & 16523 & 1.13 & 1.59 & 2.3 & 10476 & 0.93 & 1.25 & 1.75 \\
\hline & $60-70$ & 6431 & 0.99 & 1.36 & 1.9 & 4740 & 1.03 & 1.37 & 1.9 \\
\hline & $70-80$ & 3768 & 0.94 & 1.25 & 1.72 & 2305 & 1.07 & 1.41 & 1.91 \\
\hline & $>80$ & 1429 & 0.86 & 1.12 & 1.56 & 653 & 1 & 1.31 & 1.79 \\
\hline \multirow[t]{7}{*}{2016} & $<30$ & 11509 & 0.79 & 1.11 & 1.63 & 13160 & 0.58 & 0.74 & 0.96 \\
\hline & $30-40$ & 22158 & 1 & 1.45 & 2.13 & 18756 & 0.63 & 0.82 & 1.12 \\
\hline & $40-50$ & 25431 & 1.11 & 1.58 & 2.34 & 19691 & 0.73 & 0.96 & 1.34 \\
\hline & $50-60$ & 17979 & 1.09 & 1.55 & 2.24 & 11619 & 0.89 & 1.2 & 1.67 \\
\hline & $60-70$ & 7462 & 0.94 & 1.31 & 1.85 & 5050 & 0.98 & 1.33 & 1.83 \\
\hline & $70-80$ & 3776 & 0.9 & 1.21 & 1.66 & 2311 & 1.05 & 1.4 & 1.87 \\
\hline & $>80$ & 1552 & 0.82 & 1.12 & 1.56 & 681 & 0.99 & 1.3 & 1.76 \\
\hline \multirow[t]{7}{*}{2017} & $<30$ & 11697 & 0.83 & 1.15 & 1.69 & 13699 & 0.6 & 0.77 & 1.02 \\
\hline & $30-40$ & 23436 & 1.03 & 1.47 & 2.19 & 21350 & 0.66 & 0.85 & 1.15 \\
\hline & $40-50$ & 25384 & 1.14 & 1.64 & 2.43 & 20324 & 0.75 & 0.99 & 1.37 \\
\hline & $50-60$ & 18153 & 1.13 & 1.59 & 2.3 & 12811 & 0.91 & 1.24 & 1.71 \\
\hline & $60-70$ & 7614 & 0.99 & 1.35 & 1.9 & 5602 & 1 & 1.36 & 1.87 \\
\hline & $70-80$ & 3668 & 0.92 & 1.24 & 1.73 & 2401 & 1.05 & 1.39 & 1.89 \\
\hline & $>80$ & 1569 & 0.87 & 1.15 & 1.6 & 791 & 1 & 1.29 & 1.77 \\
\hline
\end{tabular}

Table 4-3 The characteristics of LDL-C among the health examination population in different ages (2009-2017). 


\begin{tabular}{|c|c|c|c|c|c|c|c|c|c|}
\hline & & Male & & & & Female & & & \\
\hline YEAR & AGE & $\mathrm{n}$ & p25 & median & p75 & $\mathrm{n}$ & p25 & median & p75 \\
\hline \multirow[t]{7}{*}{2009} & $<30$ & 3335 & 2.07 & 2.5 & 2.96 & 3365 & 1.82 & 2.16 & 2.54 \\
\hline & $30-40$ & 6015 & 2.3 & 2.78 & 3.25 & 4540 & 1.96 & 2.33 & 2.72 \\
\hline & $40-50$ & 6843 & 2.43 & 2.9 & 3.4 & 4881 & 2.2 & 2.62 & 3.08 \\
\hline & $50-60$ & 4529 & 2.48 & 2.96 & 3.47 & 2858 & 2.52 & 2.99 & 3.55 \\
\hline & $60-70$ & 2410 & 2.48 & 2.96 & 3.46 & 1494 & 2.59 & 3.14 & 3.62 \\
\hline & $70-80$ & 1666 & 2.41 & 2.89 & 3.47 & 902 & 2.63 & 3.13 & 3.66 \\
\hline & $>80$ & 420 & 2.31 & 2.83 & 3.36 & 158 & 2.47 & 3.04 & 3.71 \\
\hline \multirow[t]{7}{*}{2010} & $<30$ & 4768 & 2.07 & 2.51 & 2.99 & 5132 & 1.82 & 2.16 & 2.54 \\
\hline & $30-40$ & 8386 & 2.3 & 2.76 & 3.25 & 6794 & 1.95 & 2.32 & 2.73 \\
\hline & $40-50$ & 9917 & 2.41 & 2.88 & 3.38 & 7417 & 2.17 & 2.59 & 3.05 \\
\hline & $50-60$ & 5761 & 2.45 & 2.94 & 3.45 & 3724 & 2.52 & 2.99 & 3.51 \\
\hline & $60-70$ & 2964 & 2.43 & 2.89 & 3.43 & 1946 & 2.6 & 3.12 & 3.64 \\
\hline & $70-80$ & 2055 & 2.37 & 2.86 & 3.38 & 954 & 2.5 & 3.11 & 3.65 \\
\hline & $>80$ & 627 & 2.21 & 2.73 & 3.28 & 181 & 2.43 & 2.94 & 3.58 \\
\hline \multirow[t]{7}{*}{2011} & $<30$ & 4692 & 2.05 & 2.47 & 2.96 & 5014 & 1.8 & 2.13 & 2.54 \\
\hline & $30-40$ & 8683 & 2.27 & 2.73 & 3.22 & 7113 & 1.93 & 2.31 & 2.73 \\
\hline & $40-50$ & 11502 & 2.39 & 2.88 & 3.39 & 8259 & 2.18 & 2.6 & 3.06 \\
\hline & $50-60$ & 6378 & 2.44 & 2.92 & 3.44 & 4242 & 2.54 & 3.01 & 3.54 \\
\hline & $60-70$ & 3164 & 2.4 & 2.88 & 3.38 & 2211 & 2.59 & 3.12 & 3.66 \\
\hline & $70-80$ & 2278 & 2.35 & 2.85 & 3.37 & 1421 & 2.47 & 3.07 & 3.64 \\
\hline & $>80$ & 787 & 2.24 & 2.77 & 3.29 & 293 & 2.36 & 2.94 & 3.6 \\
\hline \multirow[t]{7}{*}{2012} & $<30$ & 5756 & 2.15 & 2.6 & 3.09 & 6736 & 1.88 & 2.24 & 2.64 \\
\hline & $30-40$ & 10362 & 2.4 & 2.87 & 3.37 & 8305 & 2.01 & 2.4 & 2.84 \\
\hline & $40-50$ & 13318 & 2.48 & 2.99 & 3.52 & 9728 & 2.26 & 2.68 & 3.16 \\
\hline & $50-60$ & 7065 & 2.54 & 3.03 & 3.54 & 4490 & 2.62 & 3.09 & 3.63 \\
\hline & $60-70$ & 3314 & 2.46 & 2.96 & 3.46 & 2185 & 2.65 & 3.19 & 3.71 \\
\hline & $70-80$ & 2375 & 2.39 & 2.88 & 3.4 & 1323 & 2.5 & 3.09 & 3.69 \\
\hline & $>80$ & 869 & 2.17 & 2.7 & 3.31 & 311 & 2.33 & 2.89 & 3.45 \\
\hline \multirow[t]{7}{*}{2013} & $<30$ & 6307 & 2.19 & 2.63 & 3.13 & 7327 & 1.9 & 2.27 & 2.68 \\
\hline & $30-40$ & 11869 & 2.41 & 2.88 & 3.38 & 9931 & 2.03 & 2.4 & 2.84 \\
\hline & $40-50$ & 14940 & 2.51 & 3 & 3.54 & 11376 & 2.28 & 2.71 & 3.19 \\
\hline & $50-60$ & 9415 & 2.53 & 3.05 & 3.55 & 6287 & 2.62 & 3.1 & 3.65 \\
\hline & $60-70$ & 4295 & 2.49 & 2.99 & 3.49 & 3034 & 2.65 & 3.18 & 3.75 \\
\hline & $70-80$ & 3069 & 2.37 & 2.91 & 3.45 & 1952 & 2.47 & 3.07 & 3.7 \\
\hline & $>80$ & 1129 & 2.16 & 2.74 & 3.35 & 444 & 2.29 & 3.03 & 3.58 \\
\hline \multirow[t]{6}{*}{2014} & $<30$ & 4752 & 2.14 & 2.58 & 3.06 & 5592 & 1.87 & 2.21 & 2.6 \\
\hline & $30-40$ & 8986 & 2.35 & 2.81 & 3.3 & 7878 & 1.99 & 2.37 & 2.8 \\
\hline & $40-50$ & 10553 & 2.44 & 2.92 & 3.43 & 8038 & 2.2 & 2.64 & 3.11 \\
\hline & $50-60$ & 7181 & 2.47 & 2.95 & 3.48 & 5051 & 2.57 & 3.05 & 3.56 \\
\hline & $60-70$ & 3509 & 2.41 & 2.94 & 3.43 & 2633 & 2.51 & 3.04 & 3.57 \\
\hline & $70-80$ & 2201 & 2.33 & 2.82 & 3.35 & 1368 & 2.47 & 3.02 & 3.59 \\
\hline
\end{tabular}

Page 12/18 


\begin{tabular}{|c|c|c|c|c|c|c|c|c|c|}
\hline & $>80$ & 929 & 2.09 & 2.73 & 3.27 & 395 & 2.23 & 2.92 & 3.49 \\
\hline \multirow[t]{7}{*}{2015} & $<30$ & 11113 & 2.1 & 2.54 & 3.01 & 13104 & 1.82 & 2.16 & 2.56 \\
\hline & $30-40$ & 20778 & 2.28 & 2.72 & 3.19 & 16958 & 1.94 & 2.3 & 2.73 \\
\hline & $40-50$ & 24381 & 2.37 & 2.83 & 3.32 & 18354 & 2.14 & 2.55 & 3.01 \\
\hline & $50-60$ & 16531 & 2.38 & 2.85 & 3.35 & 10476 & 2.45 & 2.92 & 3.43 \\
\hline & $60-70$ & 6431 & 2.31 & 2.79 & 3.29 & 4740 & 2.45 & 2.97 & 3.49 \\
\hline & $70-80$ & 3768 & 2.19 & 2.69 & 3.21 & 2306 & 2.27 & 2.85 & 3.42 \\
\hline & $>80$ & 1429 & 1.99 & 2.52 & 3.09 & 653 & 2.12 & 2.74 & 3.3 \\
\hline \multirow[t]{7}{*}{2016} & $<30$ & 11509 & 2.1 & 2.53 & 2.99 & 13160 & 1.82 & 2.15 & 2.55 \\
\hline & $30-40$ & 22158 & 2.3 & 2.75 & 3.22 & 18756 & 1.94 & 2.3 & 2.72 \\
\hline & $40-50$ & 25432 & 2.38 & 2.84 & 3.33 & 19691 & 2.16 & 2.56 & 3.02 \\
\hline & $50-60$ & 17980 & 2.4 & 2.88 & 3.36 & 11619 & 2.5 & 2.96 & 3.47 \\
\hline & $60-70$ & 7462 & 2.33 & 2.81 & 3.32 & 5050 & 2.51 & 3.02 & 3.54 \\
\hline & $70-80$ & 3776 & 2.19 & 2.71 & 3.24 & 2311 & 2.36 & 2.91 & 3.48 \\
\hline & $>80$ & 1552 & 2 & 2.56 & 3.12 & 681 & 2.21 & 2.82 & 3.36 \\
\hline \multirow[t]{7}{*}{2017} & $<30$ & 11698 & 2.2 & 2.65 & 3.15 & 13700 & 1.93 & 2.29 & 2.71 \\
\hline & $30-40$ & 23436 & 2.42 & 2.87 & 3.37 & 21350 & 2.04 & 2.42 & 2.86 \\
\hline & $40-50$ & 25387 & 2.48 & 2.97 & 3.48 & 20324 & 2.27 & 2.69 & 3.17 \\
\hline & $50-60$ & 18154 & 2.49 & 2.99 & 3.5 & 12812 & 2.6 & 3.07 & 3.6 \\
\hline & $60-70$ & 7614 & 2.45 & 2.97 & 3.49 & 5602 & 2.59 & 3.13 & 3.67 \\
\hline & $70-80$ & 3668 & 2.29 & 2.83 & 3.38 & 2401 & 2.34 & 2.99 & 3.56 \\
\hline & $>80$ & 1568 & 2.08 & 2.65 & 3.22 & 791 & 2.16 & 2.8 & 3.45 \\
\hline
\end{tabular}

Table 4-4 The characteristics of HDL-C among the health examination population in different ages (2009-2017). 


\begin{tabular}{|c|c|c|c|c|c|c|c|c|c|}
\hline & & Male & & & & Female & & & \\
\hline YEAR & AGE & $\mathrm{n}$ & p25 & median & p75 & $\mathrm{n}$ & p25 & median & p75 \\
\hline \multirow[t]{7}{*}{2009} & $<30$ & 3335 & 1.14 & 1.31 & 1.52 & 3365 & 1.45 & 1.66 & 1.88 \\
\hline & $30-40$ & 6016 & 1.06 & 1.25 & 1.46 & 4540 & 1.4 & 1.62 & 1.86 \\
\hline & $40-50$ & 6843 & 1.05 & 1.22 & 1.44 & 4881 & 1.37 & 1.6 & 1.84 \\
\hline & $50-60$ & 4530 & 1.08 & 1.26 & 1.48 & 2858 & 1.35 & 1.6 & 1.88 \\
\hline & $60-70$ & 2410 & 1.12 & 1.32 & 1.56 & 1494 & 1.31 & 1.53 & 1.81 \\
\hline & $70-80$ & 1666 & 1.16 & 1.4 & 1.67 & 902 & 1.32 & 1.56 & 1.86 \\
\hline & $>80$ & 420 & 1.25 & 1.49 & 1.8 & 158 & 1.42 & 1.68 & 2.01 \\
\hline \multirow[t]{7}{*}{2010} & $<30$ & 4768 & 1.15 & 1.34 & 1.55 & 5132 & 1.48 & 1.69 & 1.92 \\
\hline & $30-40$ & 8386 & 1.11 & 1.29 & 1.51 & 6794 & 1.44 & 1.67 & 1.92 \\
\hline & $40-50$ & 9919 & 1.09 & 1.27 & 1.49 & 7417 & 1.41 & 1.64 & 1.9 \\
\hline & $50-60$ & 5761 & 1.12 & 1.3 & 1.54 & 3724 & 1.39 & 1.66 & 1.96 \\
\hline & $60-70$ & 2964 & 1.16 & 1.37 & 1.63 & 1946 & 1.35 & 1.58 & 1.88 \\
\hline & $70-80$ & 2055 & 1.2 & 1.42 & 1.72 & 954 & 1.32 & 1.6 & 1.89 \\
\hline & $>80$ & 627 & 1.24 & 1.53 & 1.83 & 181 & 1.43 & 1.66 & 2 \\
\hline \multirow[t]{7}{*}{2011} & $<30$ & 4693 & 1.18 & 1.38 & 1.6 & 5014 & 1.54 & 1.77 & 2.01 \\
\hline & $30-40$ & 8683 & 1.13 & 1.32 & 1.56 & 7113 & 1.49 & 1.73 & 1.99 \\
\hline & $40-50$ & 11502 & 1.12 & 1.3 & 1.53 & 8259 & 1.45 & 1.69 & 1.96 \\
\hline & $50-60$ & 6378 & 1.15 & 1.34 & 1.59 & 4242 & 1.44 & 1.7 & 2 \\
\hline & $60-70$ & 3164 & 1.2 & 1.41 & 1.67 & 2211 & 1.41 & 1.66 & 1.95 \\
\hline & $70-80$ & 2278 & 1.23 & 1.47 & 1.77 & 1421 & 1.41 & 1.67 & 2 \\
\hline & $>80$ & 787 & 1.28 & 1.53 & 1.85 & 293 & 1.49 & 1.76 & 2.09 \\
\hline \multirow[t]{7}{*}{2012} & $<30$ & 5756 & 1.14 & 1.33 & 1.55 & 6736 & 1.49 & 1.71 & 1.95 \\
\hline & $30-40$ & 10362 & 1.09 & 1.28 & 1.5 & 8305 & 1.43 & 1.66 & 1.92 \\
\hline & $40-50$ & 13318 & 1.08 & 1.26 & 1.49 & 9728 & 1.42 & 1.66 & 1.92 \\
\hline & $50-60$ & 7065 & 1.11 & 1.3 & 1.52 & 4490 & 1.41 & 1.66 & 1.95 \\
\hline & $60-70$ & 3314 & 1.15 & 1.35 & 1.62 & 2185 & 1.35 & 1.61 & 1.91 \\
\hline & $70-80$ & 2376 & 1.18 & 1.42 & 1.72 & 1323 & 1.38 & 1.64 & 1.96 \\
\hline & $>80$ & 869 & 1.26 & 1.51 & 1.81 & 311 & 1.45 & 1.78 & 2.08 \\
\hline \multirow[t]{7}{*}{2013} & $<30$ & 6307 & 1.14 & 1.33 & 1.55 & 7327 & 1.47 & 1.7 & 1.94 \\
\hline & $30-40$ & 11869 & 1.07 & 1.26 & 1.48 & 9931 & 1.43 & 1.65 & 1.91 \\
\hline & $40-50$ & 14940 & 1.07 & 1.25 & 1.47 & 11376 & 1.41 & 1.65 & 1.91 \\
\hline & $50-60$ & 9415 & 1.09 & 1.29 & 1.52 & 6287 & 1.4 & 1.65 & 1.94 \\
\hline & $60-70$ & 4295 & 1.16 & 1.35 & 1.6 & 3034 & 1.35 & 1.6 & 1.89 \\
\hline & $70-80$ & 3069 & 1.17 & 1.4 & 1.69 & 1952 & 1.39 & 1.65 & 1.96 \\
\hline & $>80$ & 1129 & 1.24 & 1.49 & 1.81 & 444 & 1.43 & 1.74 & 2.1 \\
\hline \multirow[t]{6}{*}{2014} & $<30$ & 4752 & 1.15 & 1.36 & 1.58 & 5591 & 1.5 & 1.75 & 1.99 \\
\hline & $30-40$ & 8987 & 1.09 & 1.28 & 1.51 & 7878 & 1.46 & 1.7 & 1.96 \\
\hline & $40-50$ & 10554 & 1.1 & 1.29 & 1.52 & 8038 & 1.45 & 1.7 & 1.98 \\
\hline & $50-60$ & 7181 & 1.12 & 1.32 & 1.56 & 5051 & 1.43 & 1.7 & 1.99 \\
\hline & $60-70$ & 3509 & 1.18 & 1.39 & 1.64 & 2633 & 1.38 & 1.64 & 1.93 \\
\hline & $70-80$ & 2201 & 1.19 & 1.42 & 1.71 & 1368 & 1.37 & 1.64 & 1.97 \\
\hline
\end{tabular}

Page 14/18 


\begin{tabular}{|c|c|c|c|c|c|c|c|c|c|}
\hline & $>80$ & 929 & 1.29 & 1.53 & 1.84 & 395 & 1.43 & 1.72 & 2.08 \\
\hline \multirow[t]{7}{*}{2015} & $<30$ & 11113 & 1.13 & 1.33 & 1.56 & 13102 & 1.48 & 1.72 & 1.98 \\
\hline & $30-40$ & 20778 & 1.08 & 1.27 & 1.5 & 16957 & 1.43 & 1.69 & 1.95 \\
\hline & $40-50$ & 24381 & 1.08 & 1.27 & 1.52 & 18354 & 1.42 & 1.68 & 1.95 \\
\hline & $50-60$ & 16528 & 1.11 & 1.3 & 1.55 & 10476 & 1.42 & 1.68 & 1.98 \\
\hline & $60-70$ & 6431 & 1.18 & 1.39 & 1.67 & 4739 & 1.38 & 1.65 & 1.95 \\
\hline & $70-80$ & 3768 & 1.19 & 1.43 & 1.71 & 2306 & 1.39 & 1.69 & 2.02 \\
\hline & $>80$ & 1429 & 1.27 & 1.54 & 1.88 & 653 & 1.45 & 1.76 & 2.12 \\
\hline \multirow[t]{7}{*}{2016} & $<30$ & 11509 & 1.08 & 1.27 & 1.49 & 13160 & 1.43 & 1.65 & 1.9 \\
\hline & $30-40$ & 22158 & 1.02 & 1.2 & 1.42 & 18755 & 1.37 & 1.6 & 1.86 \\
\hline & $40-50$ & 25432 & 1.02 & 1.21 & 1.43 & 19691 & 1.36 & 1.6 & 1.87 \\
\hline & $50-60$ & 17979 & 1.04 & 1.23 & 1.46 & 11618 & 1.34 & 1.59 & 1.88 \\
\hline & $60-70$ & 7461 & 1.1 & 1.31 & 1.57 & 5050 & 1.31 & 1.56 & 1.85 \\
\hline & $70-80$ & 3776 & 1.11 & 1.34 & 1.61 & 2311 & 1.3 & 1.56 & 1.87 \\
\hline & $>80$ & 1552 & 1.18 & 1.41 & 1.72 & 681 & 1.36 & 1.64 & 1.99 \\
\hline \multirow[t]{7}{*}{2017} & $<30$ & 11698 & 1.06 & 1.25 & 1.46 & 13699 & 1.41 & 1.64 & 1.88 \\
\hline & $30-40$ & 23436 & 1.01 & 1.19 & 1.4 & 21348 & 1.35 & 1.58 & 1.84 \\
\hline & $40-50$ & 25386 & 1.01 & 1.19 & 1.41 & 20321 & 1.35 & 1.59 & 1.86 \\
\hline & $50-60$ & 18153 & 1.03 & 1.21 & 1.43 & 12809 & 1.34 & 1.59 & 1.87 \\
\hline & $60-70$ & 7611 & 1.09 & 1.3 & 1.55 & 5601 & 1.31 & 1.56 & 1.85 \\
\hline & $70-80$ & 3668 & 1.11 & 1.33 & 1.6 & 2401 & 1.28 & 1.53 & 1.85 \\
\hline & $>80$ & 1569 & 1.15 & 1.38 & 1.68 & 790 & 1.35 & 1.63 & 1.98 \\
\hline
\end{tabular}

\section{Figures}




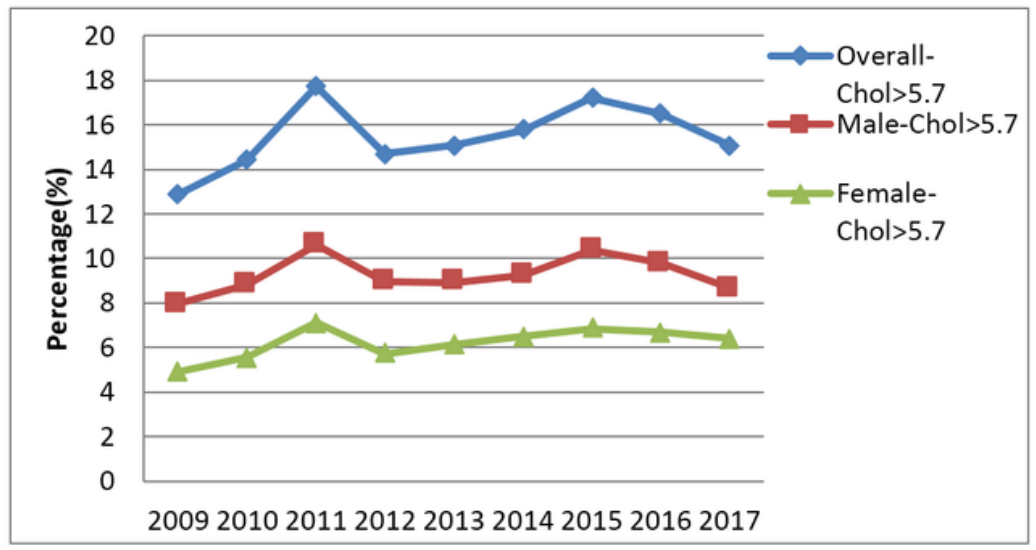

\section{A}

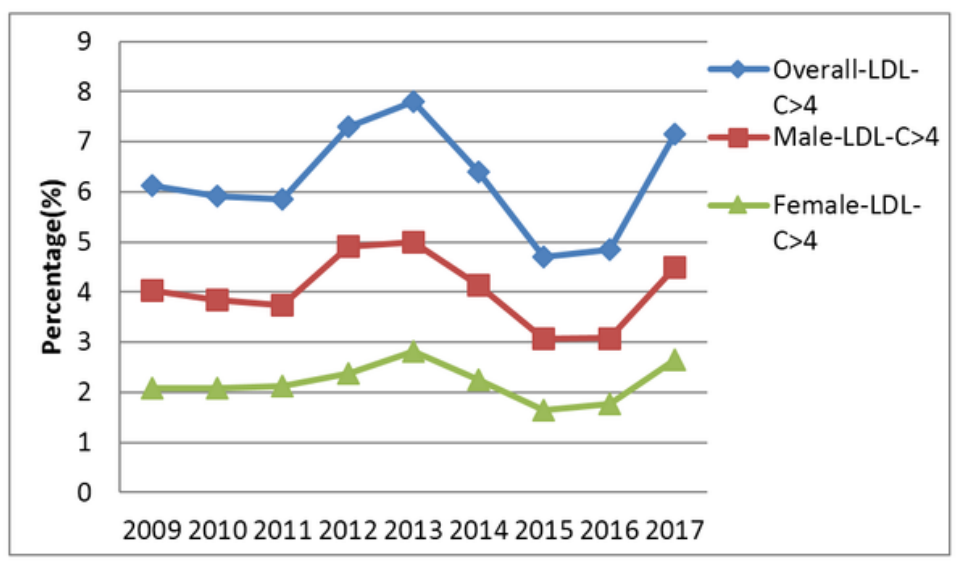

C

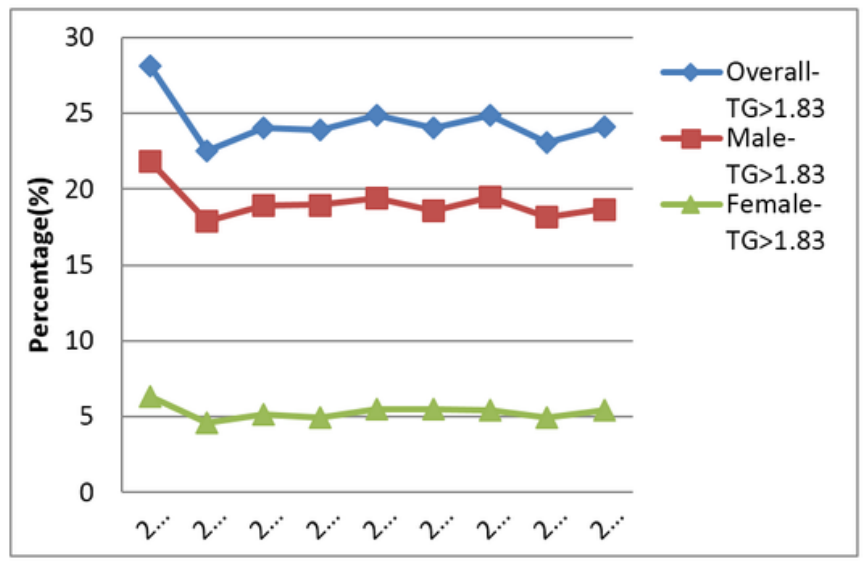

B

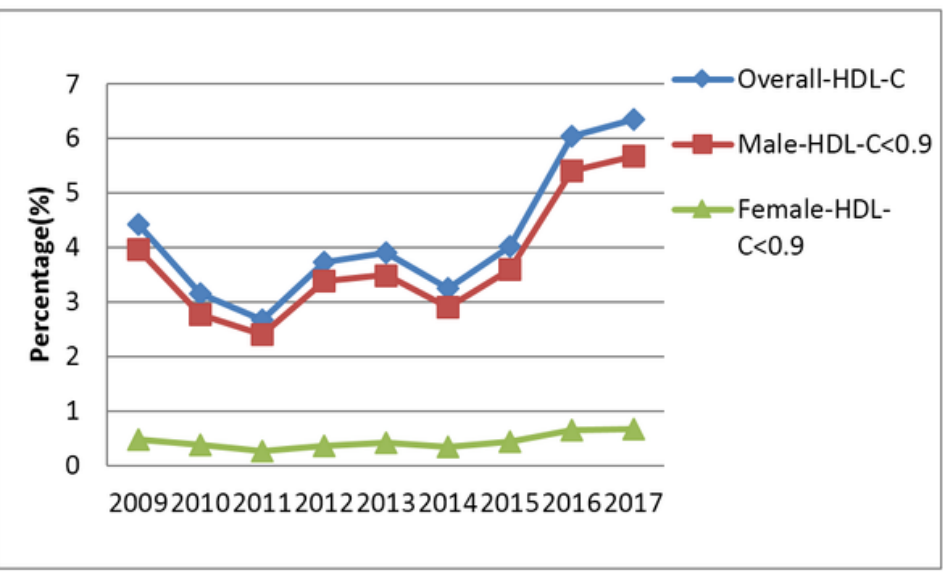

D

Figure 1

A. Trends of the percentage of people with blood cholesterol levels of more than $5.7 \mathrm{mmol} / \mathrm{L}$ in both genders(2009-2017). B. Trends of the percentage of people with blood triglyceride levels of more than $1.83 \mathrm{mmol} / \mathrm{L}$ in both genders(2009-2017). C. Trends of the percentage of people with blood LDL-C levels of more than $4.0 \mathrm{mmol} / \mathrm{L}$ in both genders(2009-2017). D. Trends of the percentage of people with blood HDL-C levels of less than $0.9 \mathrm{mmol} / \mathrm{L}$ in both genders(2009-2017). 


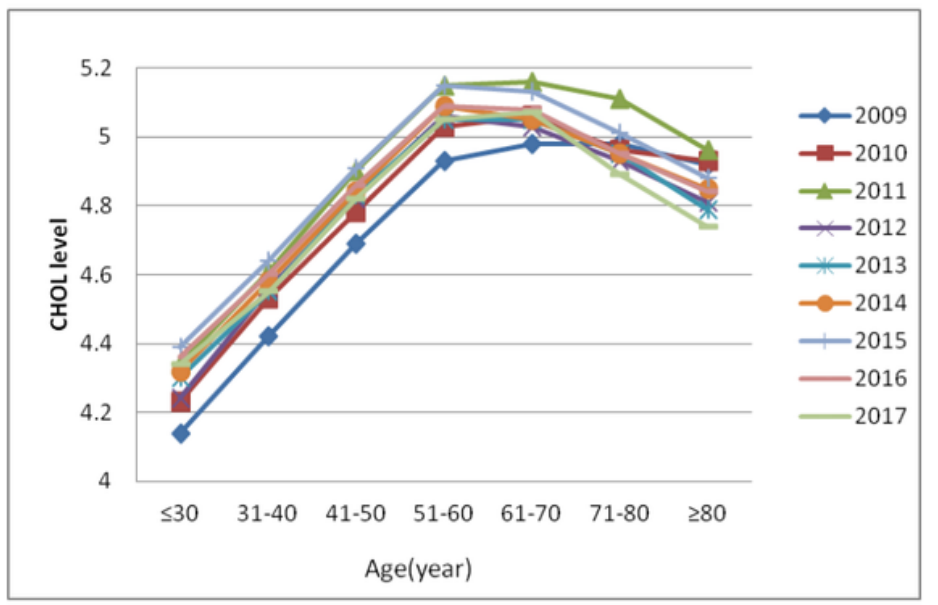

A

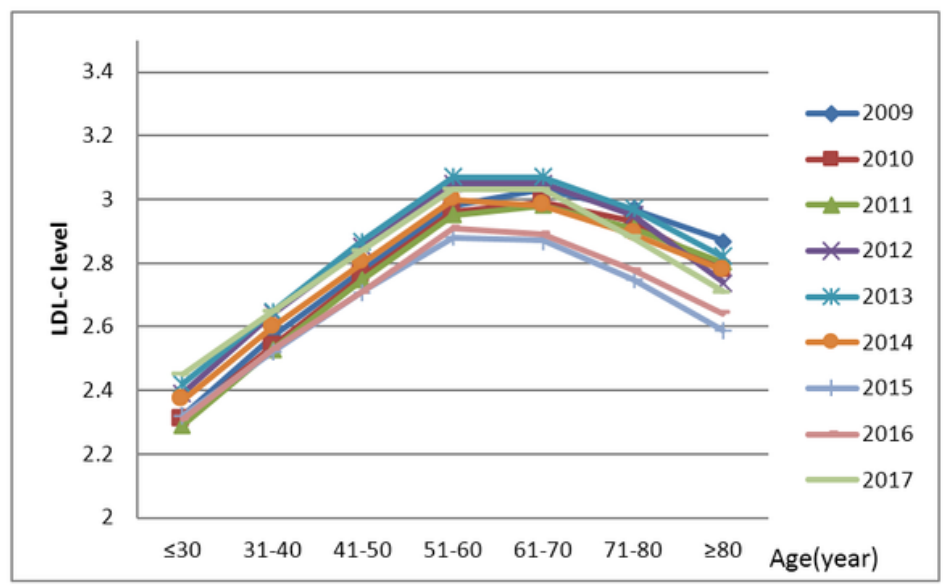

C

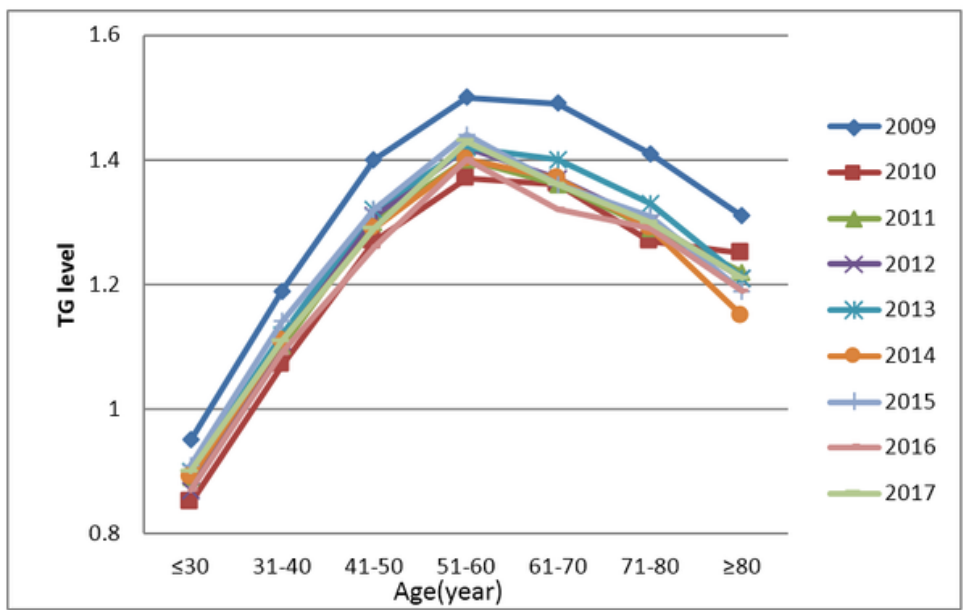

B

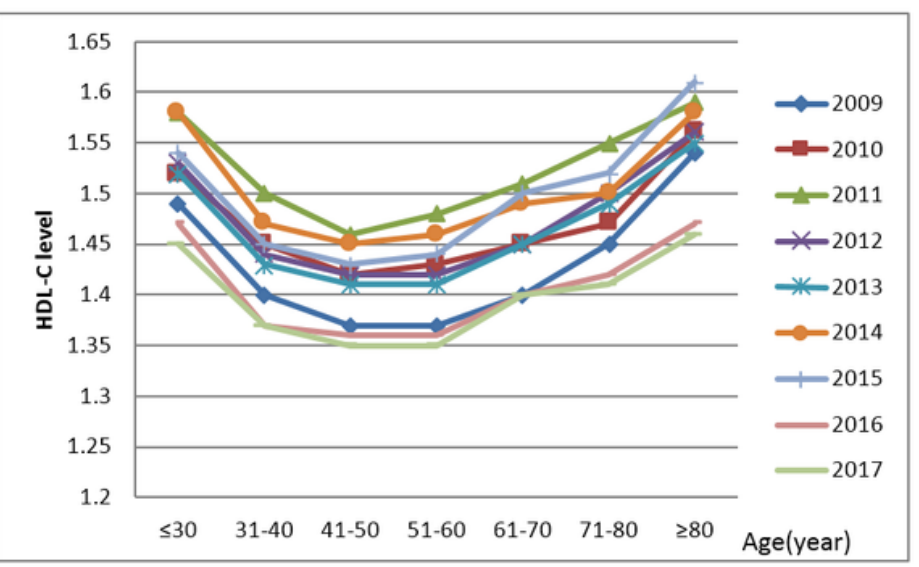

D

Figure 2

A. The tendency of cholesterol levels in different age groups among different years. B. The tendency of triglyceride levels in different age groups among different years. C. The tendency of LDL-C levels in different age groups among different years. D. The tendency of HDL-C levels in different age groups among different years. 


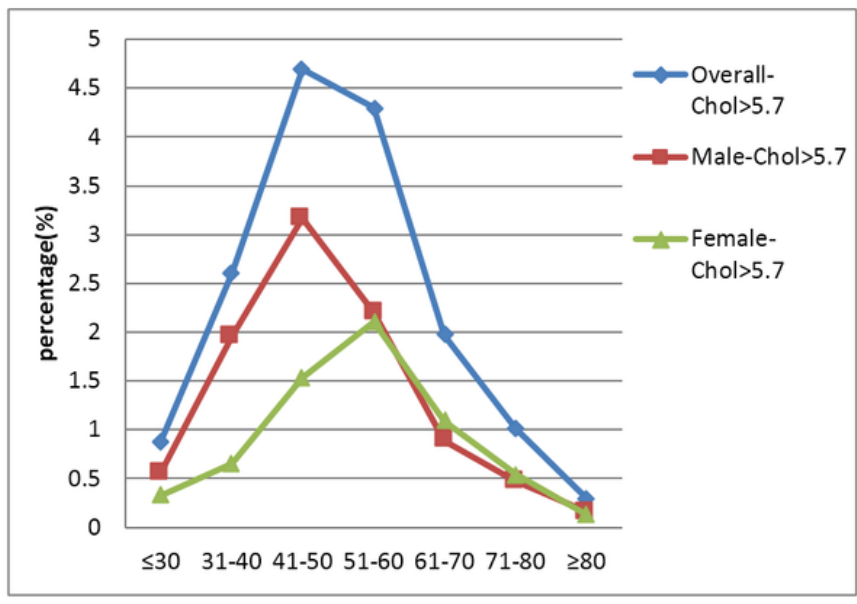

A

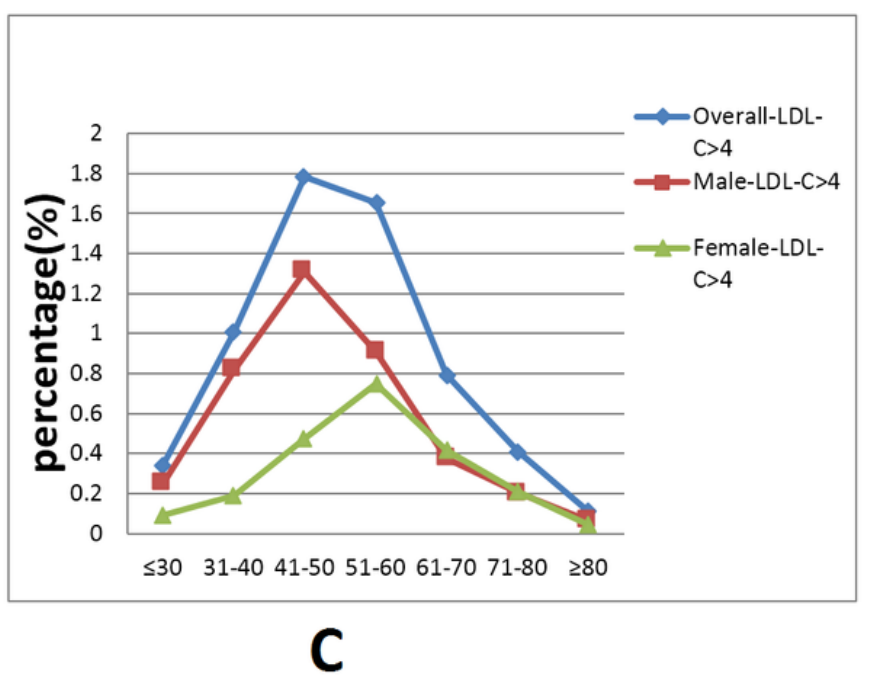

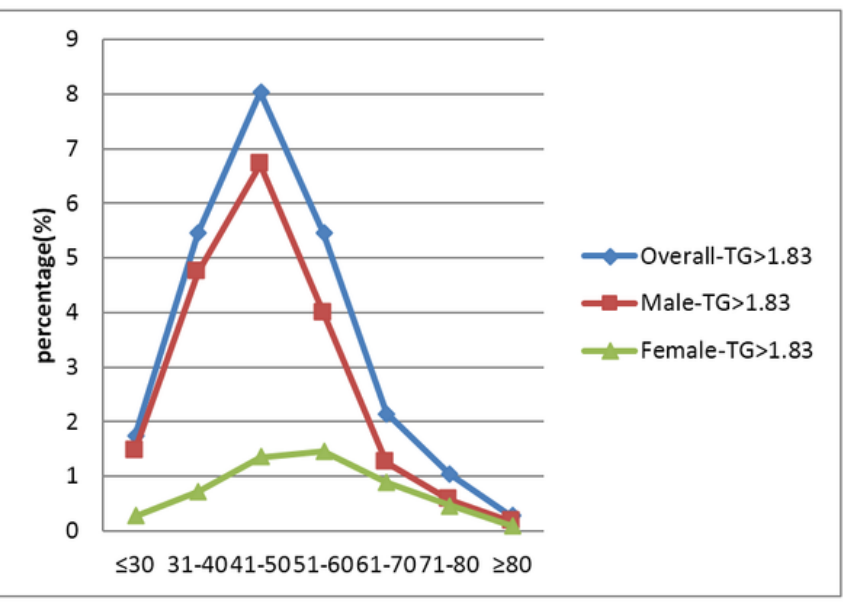

B

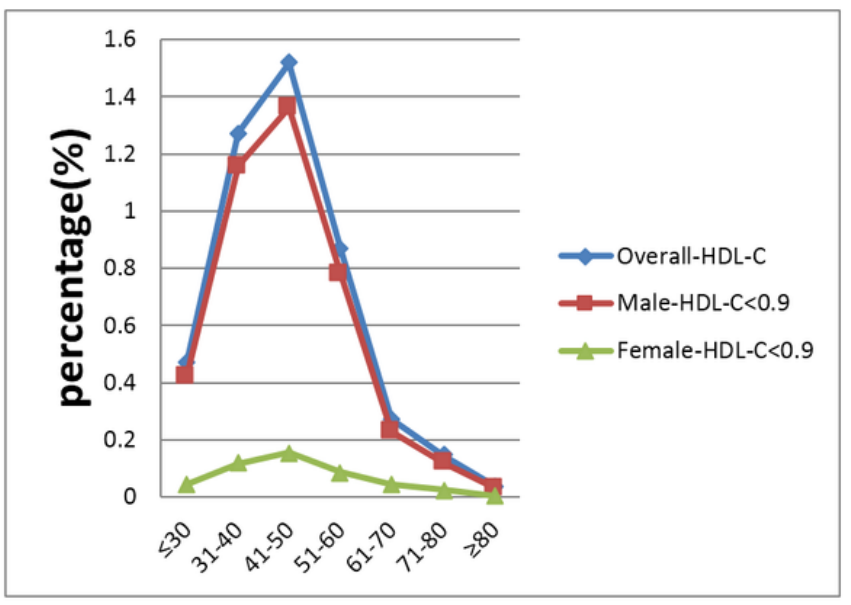

D

Figure 3

A. Trends of the percentage of people with blood cholesterol levels of more than $5.7 \mathrm{mmol} / \mathrm{L}$ in different age groups. B. Trends of the percentage of people with blood triglyceride levels of more than $1.83 \mathrm{mmol} / \mathrm{L}$ in different age groups. C. Trends of the percentage of people with blood LDL-C levels of more than $4.0 \mathrm{mmol} / \mathrm{L}$ in different age groups. D. Trends of the percentage of people with blood HDL-C levels of less than $0.9 \mathrm{mmol} / \mathrm{L}$ in different age groups. 